\title{
Primary Amebic Meningoencephalitis: A Case Report
}

\author{
Minhua Chen', Wei Ruan², Lingling Zhang'2, Bangchuan $\mathrm{Hu}^{1, *}$, Xianghong Yang ${ }^{1}$ \\ ${ }^{1}$ Department of Critical Care Medicine, Zhejiang Provincial People's Hospital, People's Hospital of Hangzhou Medical College, Hangzhou 310014, \\ Zhejiang, China; '2Department of Communicable Diseases of Control and Prevention, Zhejiang Provincial Centre for Disease Control and Prevention, \\ Hangzhou 310000, Zhejiang, China
}

\begin{abstract}
Primary amebic encephalitis (PAM) is a devastating central nervous system infection caused by Naegleria fowleri, a free-living amoeba, which can survive in soil and warm fresh water. Here, a 43-year-old healthy male was exposed to warm freshwater 5 days before the symptom onset. He rapidly developed severe cerebral edema before the diagnosis of PAM and was treated with intravenous conventional amphotericin B while died of terminal cerebral hernia finally. Comparing the patients with PAM who has similar clinical symptoms to those with other common types of meningoencephalitis, this infection is probably curable if treated early and aggressively. PAM should be considered in the differential diagnosis of purulent meningoencephalitis, especially in patients with recent freshwater-related activities during the hot season.
\end{abstract}

Key words: Naegleria fowleri, primary amebic meningoencephalitis, cerebral hernia, amphotericin B

\section{INTRODUCTION}

Naegleria fowleri is a free-living amoeba that can survive in soil and warm fresh water [1]. When humans are exposed to contaminated water, amoeba enters the nasal cavity of the host and travels through the nasal mucosa, followed by moving along the cribriform plate and olfactory nerve, finally reaching the olfactory bulbs. Consequently, it destroys the central nervous system (CNS), thereby causing primary amebic meningoencephalitis (PAM) [2]. Although the infection is rare, it is fatal. To date, there have been only 7 cases reported in China, and none of them survived because of a severe increase of cerebrospinal fluid (CSF) and intracranial pressure.

\section{CASE RECORD}

A 43-year-old, previously healthy male, visited the emergency department of Zhejiang Provincial People's Hospital, Hangzhou, China, on 20 August 20, 2016, with a 2-day history of persistently occipital headache, fever, myalgia, and fatigue. He was a tourist from Shenzhen, China, which is a large city lo-

- Received 22 February 2019, revised 10 May 2019, accepted 10 May 2019

*Corresponding author (hubangchuanicu@163.com)

(c) 2019, Korean Society for Parasitology and Tropical Medicine

This is an Open Access article distributed under the terms of the Creative Commons Attribution Non-Commercial License (http://creativecommons.org/licenses/by-nc/4.0) which permits unrestricted non-commercial use, distribution, and reproduction in any

medium, provided the original work is properly cited. cated in southern China. Before visiting the Hospital, he had self-administered some NSAIDs (non-steroidal anti-inflammatory drugs) without achieving any relief. The headache was excruciating and intolerable.

At the time of Hospital admission, his vital signs were as follows: temperature, $39.1^{\circ} \mathrm{C}$; pulse, 89 beats/min; respiratory rate, 33 breaths/min; blood pressure, 133/56 mmHg. Physical examination revealed the presence of positive meningeal irritation sign and left Babinski sign, while the CT scan of the head was normal. The routine blood test showed the leucocyte count of 10,480 cells $/ \mathrm{mm}^{3}$ with $80 \%$ neutrophils and C-reactive protein (CRP) of $2.6 \mathrm{mg} / \mathrm{L}$. Then, lumbar puncture was dispensed, the CSF was turbid with high pressure (300 $\mathrm{mmHg}$ ), positive Pandy's test, very low level of glucose $(<1 \mathrm{mmol} / \mathrm{L})$, highly elevated leukocytes (1,170 cells/ $\mathrm{mm}^{3}$ with $83 \%$ neutrophils) and protein (>300 mg/dl). According to the above results, the patient was diagnosed with bacterial meningitis and administered empirical treatment. On August 21, he was intubated and sedated because of tachypnea and agitation, and then, transferred to the intensive care unit (ICU).

Considering the possibility of pyogenic meningitis, he was treated with $2 \mathrm{~g}$ meropenem every $8 \mathrm{hr}, 600 \mathrm{mg}$ linezolid every $12 \mathrm{hr}$ intravenously, as well as, mannitol and dexamethasone. On the second evening of ICU stay, he suddenly developed a decreased responsiveness; the pupillary examination revealed the presence of fixed and dilated pupils. Thus, a $150 \mathrm{ml}$ of $20 \%$ mannitol was infused for $30 \mathrm{~min}$, followed by CT scan- 


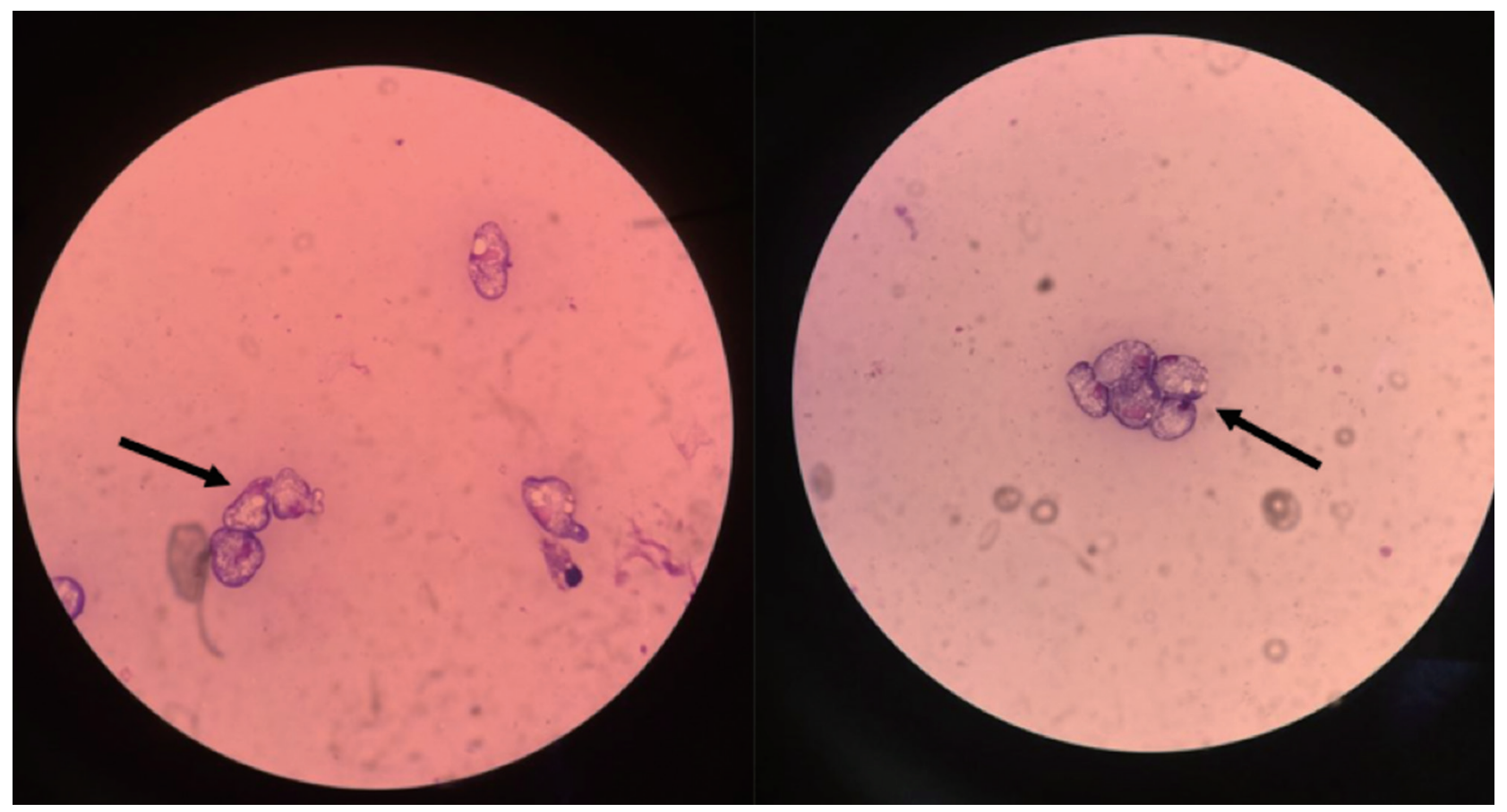

Fig. 1. Wright-Giemsa staining of CSF shows trophozoites (arrow) of N. fowleri.

ning. The brain CT scan demonstrated hydrocephalus, brain swelling, hyperdense interhemispheric fissure cistern, and supra sella cistern sign that confirmed cerebral hernia and meningoencephalitis. Subsequently, the external ventricular drainage was placed, and CSF samples were collected and reexamined. On August 24, he was eventually diagnosed with PAM as the trophozoites of $N$. fowleri were identified in the CSF via Wright-Giemsa staining (Fig. 1) and positive amplicons were obtained from the PCR for Naegleria spp. and N. fowleri with amplification fragment lengths of about $410 \mathrm{bp}$ and 310 bp, respectively (Fig. 2). His family members recollected that he had been to a water park 5 days (5d) before the onset of symptoms. Then linezolid was discontinued immediately, and regime consisting of intravenous conventional amphotericin B (5 mg initial dose followed by $10 \mathrm{mg}$ on day 2, $25 \mathrm{mg}$ on day 3, and $50 \mathrm{mg} /$ day thereafter) and fluconazole ( $400 \mathrm{mg} /$ day) was begun immediately. Nevertheless, the result of a repeat CSF study after 5 days target treatment was negative for trophozoites and his vital signs became relatively stable with normal temperature, pulse, and pressure; yet the analysis of Glasgow coma scale (GCS) continually showed 3 points and no spontaneous breathing was detected. Considering the prognosis, the patient was transferred to a local hospital in Shenzhen on 30 August, he died on 3 September.

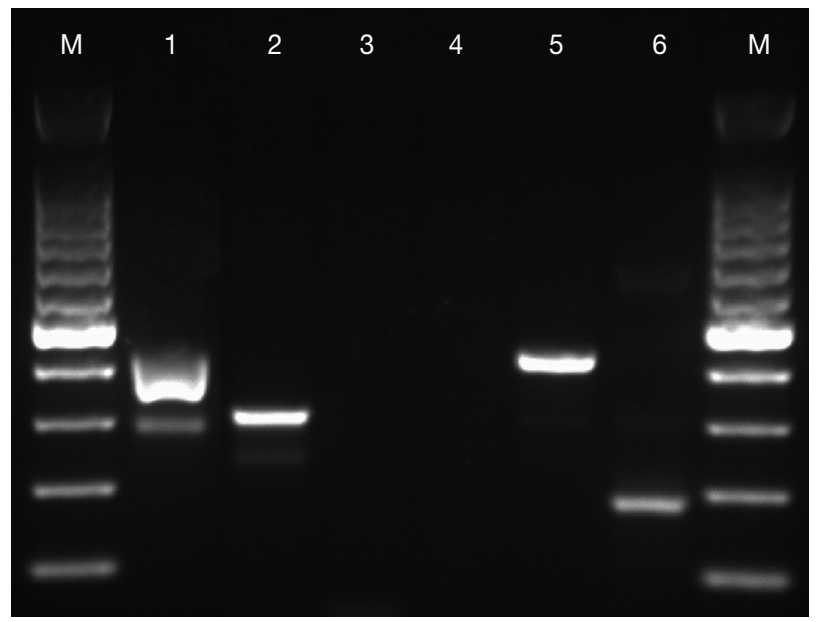

Fig. 2. PCR products revealed by agarose gel electrophoresis. M: 100-bp marker; lanes 1-6: amplified with primers of Naegleria spp., N. fowleri, Entamoeba histolytica, Entamoeba dispar, Vahlkampfia spp., and Acanthamoeba spp., respectively.

\section{DISCUSSION}

Since the first case of PAM was described by Fowler and Carter in Australia in 1965 [3], there have been nearly 200 cases reported hitherto, including 7 cases from China. Although N. fowleri is distributed worldwide, interestingly, a majority of 
the reported cases are from the USA with a total number of 138 cases until 2015 [4]. N. fowleri commonly occurs in warm freshwater; thus, any freshwater-related activities such as swimming or diving could bring the water into the nose, resulting in PAM. However, the incidence of PAM is extremely low, which indicates that exposure rarely leads to infection. Nonetheless, factors related to the occurrence of PAM are yet unknown, raising several concerns about the prevention and detection of the infections. Some theories suggest avoiding the freshwater-related activities, especially jumping into the body of water, splashing, or submerging their heads under the water during the summer season when the water temperature is high. If such activities cannot be avoided, individuals should use nose clips during the activities or rinse the nose and nasal passages with clean water immediately after such activities [5].

The fatality of PAM is extremly high with only 7 survivors to date [5]. This might be attributed to the following 2 reasons: Firstly, PAM is underdiagnosed since clinical symptoms are similar to those of other more commonly known types of meningitis [6]. Similar to this case, headache, fever, nausea, and vomiting are common clinical manifestations. The diagnosis of PAM is primarily based on the presence of trophozoites in the CSF that can be detected by trichrome or Giemsa stain. The time spent on diagnosis often results in delayed treatment. Most patients progress rapidly into a deep coma with significantly high intracranial pressure and CSF pressure before diagnosis, which can lead to death [7]. Secondly, there are no relevant clinical studies that have assessed the efficacy of a specific treatment option [5]. All the treatment methods, including the recommendation from The Centers for Disease Control and Prevention (CDC), are either based on case reports or in vitro studies. Presently, amphotericin B is accepted as the first choice drug for PAM. The N. fowleri is highly sensitive to amphotericin B in vitro [8], and all survivors from North America were reported to have received this medication $[6,9,10]$. In this case, the result of a repeat CSF study became negative for trophozoites after 5 days target treatment. The recommended therapy of CDC comprises of conventional amphotericin $\mathrm{B}$ administered intravenously at the dosages of 1.5 $\mathrm{mg} / \mathrm{kg} /$ day in 2 doses for 3 day, followed by $1 \mathrm{mg} / \mathrm{kg} /$ day for an additional 11 day. The intrathecal administration of conventional amphotericin B ( $1.5 \mathrm{mg} /$ day for 2 day and $1 \mathrm{mg} /$ day for an additional 8 day) can also be used in combination with intravenous amphotericin B [11]. Other medications that could be potentially effective include fluconazole, miconazole, miltefosine, azithromycin, and rifampin, which are usually used in combination with conventional amphotericin B [5].

Although this fatal disease might be cured if treated early and effectively, most clinicians might have never encountered it before. Therefore, it is imperative for clinicians to have an awareness of the possibility of PAM in every case of purulent meningitis, especially in patients with recent freshwater exposure during the hot season.

\section{ACKNOWLEDGMENT}

This study was financially funded by the General Project Funds from the Health Department of Zhejiang Province. Grant No. 2015KYA018.

\section{REFERENCES}

1. De Jonckheere JF. Origin and evolution of the worldwide distributed pathogenic amoeboflagellate Naegleria fowleri. Infect Genet Evol 2011; 11: 1520-1528.

2. Jarolim KL, McCosh JK, Howard MJ, John DT. A light microscopy study of the migration of Naegleria fowleri from the nasal submucosa to the central nervous system during the early stage of primary amebic meningoencephalitis in mice. J Parasitol 2000; 86: 50-55.

3. Fowler M, Carter RF. Acute pyogenic meningitis probably due to Acanthamoeba sp.: a preliminary report. Br Med J 1965; 2: 740742.

4. Cope JR, Ali IK. Primary amebic meningoencephalitis: what have we learned in the last 5 years? Curr Infect Dis Rep 2016; 18: 31-37.

5. Grace E, Asbill S, Virga K. Naegleria fowleri: Pathogenesis, Diagnosis, and Treatment Options. Antimicrob Agents Chemother 2015; 59: 6677-6681.

6. Capewell LG, Harris AM, Yoder JS, Cope JR, Eddy BA, Roy SL, Visvesvara GS, Fox LM, Beach MJ. Diagnosis, clinical course, and treatment of primary amoebic meningoencephalitis in the United States, 1937-2013. J Pediatric Infect Dis Soc 2015; 4: 68-75.

7. Visvesvara GS, Moura H, Schuster FL. Pathogenic and opportunistic free-living amoebae: Acanthamoeba spp., Balamuthia mandrillaris, Naegleria fowleri, and Sappinia diploidea. FEMS Immunol Med Microbiol 2007; 50: 1-26.

8. Carter RF. Sensitivity to amphotericin B of a Naegleria sp. related from a case of primary amoebic encephalitis. J Clin Pathol 1969; 22: 470-474.

9. Seidel JS, Harmatz P, Visvesvara GS, Cohen A, Edwards J, Turner J. Successful treatment of primary amebic meningoencephalitis. N Engl J Med 1982; 306: 346-348.

10. Vargas-Zepeda J, Gómez-Alcalá AV, Vásquez-Morales JA, LiceaAmaya L, De Jonckheere JF, Lares-Villa F. Successful treatment of 
Naegleria fowleri meningoencephalitis by using intravenous amphotericin B, fluconazole and rifampicin. Arch Med Res 2005; 36: 83-86.
11. Centers for Disease Control and Prevention. May 2014. Atlanta, USA. N. fowleri treatment. Centers for Disease Control and Prevention. 